Vol. 3, No. 2, 2017

\author{
Vasyl Olshanskiy $^{1}$, Stanislav Olshanskiy ${ }^{2}$, Maksym Slipchenko ${ }^{3}$ \\ ${ }^{1}$ Department of Physics and Theoretical Mechanics, Petro Vasilenko Kharkiv National Technical University \\ of Agriculture, 44, Artema Str., Kharkiv, Ukraine, E-mail: OlshanskiyVP@gmail.com \\ ${ }^{2}$ Department of Physics and Theoretical Mechanics, Petro Vasilenko Kharkiv National Technical University \\ of Agriculture, 44, Artema Str., Kharkiv,Ukraine, E-mail: stasolsh77@gmail.com \\ ${ }^{3}$ Department of Physics and Theoretical Mechanics, Petro Vasilenko Kharkiv National Technical University \\ of Agriculture, 44, Artema Str., Kharkiv, Ukraine, E-mail: Slipchenko_M@ukr.net
}

\title{
ON FREE OSCILLATIONS OF A QUADRATIC NONLINEAR OSCILLATOR
}

Received: October 19, 2017 / Revised: Novemver 30, 2017 / Accepted: December 26, 2017

(C) Olshanskiy V., Olshanskiy S., Slipchenko M., 2017

\begin{abstract}
A free oscillations of a system with one degree of freedom, caused either by the initial deviation from the stable equilibrium position or by the initial velocity provided by the oscillator in this position was considered. Analytical solutions of the nonlinear Cauchy problem for a second-order differential equation were constructed. The solutions are expressed in terms of Jacobi's periodic elliptic functions relating to occultation of special functions. Compact equals are derived for calculating the displacements of the oscillator and the oscillation periods for various methods of motion perturbation and for various variants of the elastic characteristic. The restrictions on the initial excitations for an oscillator with a soft elastic characteristic are determined, when its free oscillations are possible. The existence of a solution of the nonlinear dynamics problem in elementary functions is established. The behavior of an oscillator with a soft characteristic of elasticity under conditions of its freezing are studied. It is shown that from the derived equals, as special cases, the results known in the theory of linear oscillators, as well as oscillators with a purely quadratic nonlinearity, without a linear component, follow when the solution of the problem can be expressed in terms of Ateb-functions. The aim of the work was to derive new calculation equals for determining the displacements of a mechanical system with one degree of freedom under conditions of free oscillations, in the absence of friction. To achieve this objective, the representation of the second integral of the differential equation of motion due to the incomplete elliptic integral of the first kind were used. Using the known tables of the indicated integral, examples of calculations are given in which the probability of the derived equals is confirmed. According to the results of the study, it is also established that in the case of a quadratic elasticity characteristic of the linear component, the motion of the oscillator is described by the periodic elliptic Jacobi function, both in providing it with an initial deviation from the stable equilibrium position, and giving it the initial velocity in this position. In the case of a soft elasticity characteristic, free oscillations are possible only with certain restrictions on the initial perturbations of the system.
\end{abstract}

Keywords: quadratically nonlinear oscillator, soft and rigid elasticity characteristic, free oscillations, analytical solution, periodic elliptic functions.

\section{Introduction}

Due to linear oscillations with large amplitudes, emergency destruction of structural elements or premature failure of them can occur. Therefore, despite significant advances in the study of nonlinear oscillations and a fairly long history of their conduct [1], this topic is still being given considerable attention.

\section{Problem Statement}

The purpose of this article is to construct and approximate new analytical solutions describing the free oscillations of a quadratically nonlinear oscillator under two variants of the elastic characteristic. To 
achieve this goal, a method based on reducing the second integral of the differential equation of motion to an incomplete elliptic integral of the first kind is chosen. To achieve this goal, a method based on reducing the second integral of the differential equation of motion to an incomplete elliptic integral of the first kind is chosen. As a result, the displacement of the oscillator is expressed in terms of periodic Jacobi elliptic functions; therefore, for the calculation, one can use tables of these special functions or tables of an incomplete elliptic integral of the first kind. The latter are also needed to calculate the periods of oscillations. To verify the adequacy of the theoretical results obtained, limit transitions are made, by directing one of the two rigidity parameters of the oscillator to zero. Therefore, the limiting variants are either a linear oscillator or a purely quadratic one, without a linear component in the elastic response. A special rare case is also revealed when the solution of the nonlinear equation of motion is fed into elementary functions.

\section{Review of Modern Information Sources}

Not referring to numerous publications in the form of separate articles, we will single out only monograph editions of recent years [2-5], where there are corresponding reviews of literary sources. Partially, the problem of nonlinear oscillations of systems of variable mass with dry friction was also violated in [6,7], where approximate analytical methods were used for research.

\section{Main Material Presentation}

We describe the motion of an oscillator by a differential equation:

$$
m \ll \alpha+\beta x^{2} \operatorname{sign}(x)=0,
$$

where $m$ is the mass of the oscillator; $\alpha, \beta$ are characteristics of its elasticity, and $\alpha \geq 0 ; x=(t)$ is displacement of the oscillator in time $t$; the dot denotes the derivative with respect to $t$.

We will distinguish between hard $(\beta>0)$ and soft $(\beta<0)$ elasticity characteristics.

For the initial conditions for (1) we take two versions:

$$
\begin{aligned}
& \text { a) } x(0)=a ; \quad s(0)=0 ; \\
& \text { b) } x(0)=0 ; \quad\left(v_{0}\right)=v_{0} .
\end{aligned}
$$

In the first, the oscillations are caused by the initial deviation $a$ of the oscillator from the equilibrium position $x=0$, and in the second - by the initial velocity $v_{0}$ given to it in this position.

1. Free oscillations of an oscillator with a rigid characteristic of elasticity. The transition to new variables $\&=v, v \frac{d v}{d x}$, equation (1), for $(x>0)(t>0)$, we give the form:

$$
v \frac{d v}{d x}=-\frac{1}{m}\left(\alpha x+\beta x^{2}\right)
$$

Its integral, up to a constant $C$, is the expression:

$$
v^{2}=\frac{2}{m}\left(C-\frac{\alpha}{2} x^{2}+\frac{\beta}{3} x^{3}\right)
$$

For initial conditions a) in (2) the constant takes the value $C=\frac{\alpha}{2} a^{2}+\frac{\beta}{3} a^{3}$. Therefore, the speed of the oscillator is given by the equal:

$$
v=\frac{d x}{d t}=-\sqrt{\frac{2}{m}} \sqrt{\frac{\alpha}{2}\left(a^{2}-x^{2}\right)+\frac{\beta}{3}\left(a^{3}-x^{3}\right)},
$$

or

$$
\frac{d x}{d t}=-\sqrt{\frac{2 \beta}{3 m}} \sqrt{(a-x)\left[a^{2}+\frac{3 \alpha}{2 \beta} a+\left(a+\frac{3 \alpha}{2 \beta}\right) x+x^{2}\right]} .
$$


Further integration of expression (4) gives:

$$
\int^{a} \frac{d u}{(a-u)\left[\left(u+\frac{1}{2}\left(a+\frac{3 \alpha}{2 \beta}\right)\right)^{2}+\frac{3}{4}\left(a^{2}+\frac{\alpha a}{\beta}-\frac{3}{4} \frac{\alpha^{2}}{\beta^{2}}\right)\right]}=\sqrt{\frac{2 \beta}{3 m}} t .
$$

In calculating this integral, we distinguish three cases. Consider the first of these, corresponds to small oscillation amplitudes, or weak nonlinearity, when $a^{2}+\frac{\alpha a}{\beta}-\frac{3}{4} \frac{\alpha^{2}}{\beta^{2}}<0$. Then the sum in square brackets under the root can be submitted in the form of a product, $(u+b)(u+d)$ and:

So the expression (5) takes the form:

$$
\begin{aligned}
& b=\frac{1}{2}\left(a+\frac{3 \alpha}{2 \beta}\right)+\sqrt{\frac{3}{4}\left(\frac{3}{4} \frac{\alpha^{2}}{\beta^{2}}-a^{2}-\frac{\alpha a}{\beta}\right)} ; \\
& d=\frac{1}{2}\left(a+\frac{3 \alpha}{2 \beta}\right)-\sqrt{\frac{3}{4}\left(\frac{3}{4} \frac{\alpha^{2}}{\beta^{2}}-a^{2}-\frac{\alpha a}{\beta}\right)} .
\end{aligned}
$$

$$
\int_{x}^{a} \frac{d u}{\sqrt{(\alpha-u)(u+b)(u+d)}}=\sqrt{\frac{2 \beta}{3 m}} t
$$

and its left part reduces to an incomplete elliptic integral of the first kind $F(\varphi, k)$ [8]. Therefore, instead of (6), we get:

$$
F(\varphi, k)=\frac{\sqrt{a+b}}{2} \sqrt{\frac{2 \beta}{3 m}} t=\tau,
$$

where $k=\sqrt{\frac{a+d}{a+b}} ; \varphi=\arcsin \sqrt{\frac{a-x}{a+d}}=\operatorname{am}(\tau, k)$.

From the last expression it follows that:

$$
\frac{x}{\alpha}=1-\left(1+\frac{d}{a}\right) s n^{2}(\tau, k),
$$

where $\operatorname{sn}(\tau, k)=\sin [\operatorname{am}(\tau, k)]-$ Jacobi's elliptical sine $[9,10]$. Let's carry out the passage to the limit in (8) for $\beta \rightarrow 0$. For such a variation of the system: $d \rightarrow a ; b \rightarrow \infty ; \sqrt{a+b} \sqrt{\beta} \rightarrow \sqrt{\frac{3 \alpha}{2}} ; \tau \rightarrow \frac{1}{2} \sqrt{\frac{\alpha}{m}} t$; $k \rightarrow 0 ; \operatorname{sn}(\tau, k) \rightarrow \sin \tau$ and equal (8) takes the form:

$$
\frac{x}{\alpha}=1-2 \sin ^{2}\left(\frac{1}{2} \sqrt{\frac{\alpha}{m}} t\right)=\cos \sqrt{\frac{\alpha}{m}} t,
$$

which agrees with the theory of linear oscillators.

Dependences (7) make it possible to derive a equal for calculating the period of oscillations. If you put them in $x=0 ; \varphi=\varphi_{0}=\arcsin \sqrt{a /(a+d)} ; t=T / 4$. Then we arrive at the relation:

Therefore:

$$
F\left(\varphi_{0}, k\right)=\frac{T}{8} \sqrt{\frac{2(a+b) \beta}{3 m}} .
$$

$$
T=8 \sqrt{\frac{3 m}{2(a+b) \beta}} F\left(\varphi_{0}, k\right)
$$

and in determining the period of oscillations, tables of an incomplete elliptic integral of the first kind can be used [10]. 
If we pass to the limiting transition $\beta \rightarrow 0$ to a linear oscillator, $k \rightarrow 0$, $\varphi_{0} \rightarrow \arcsin (1 / \sqrt{2})=\pi / 4, F\left(\varphi_{0}, k\right) \rightarrow \varphi_{0}$ then, instead of (10), we obtain the well-known equal:

$$
T=2 \pi \sqrt{\frac{m}{\alpha}} .
$$

In the second case, when $\alpha^{2}+\frac{\alpha a}{\beta}-\frac{3}{4} \frac{\alpha^{2}}{\beta^{2}}<0$, we have: $a=\frac{\alpha}{2 \beta} ; b=d=2 a$. Therefore, the expression (5) takes the form of:

$$
I=\int_{x}^{a} \frac{d u}{\sqrt{a-u}(u+2 a)}=\sqrt{\frac{2 \beta}{3 m}} t .
$$

This integral is expressed in terms of elementary functions. Indeed, after making substitutions $a-u=y^{2} ; d u=-2 y d y ; u+2 a=3 a-y^{2}$, we get:

$$
I=2 \int_{0}^{\sqrt{a-x}} \frac{d y}{3 a-y^{2}}=\frac{1}{\sqrt{3 a}} \ln \frac{\sqrt{3 a}+\sqrt{a-x}}{\sqrt{3 a}-\sqrt{a-x}}=\sqrt{\frac{2 \beta}{3 m}} t .
$$

It follows that:

$$
x=a\left(1-3 \operatorname{th}^{2} \frac{\omega t}{2}\right)
$$

where $\omega=\sqrt{\frac{\alpha}{m}}$.

As we see, in the second case the solution of the nonlinear differential equation (1) is expressed in elementary functions.

For such functions, the equal for the period of oscillations reduces, because in (12):

$$
\frac{1}{\sqrt{3 a}} \ln \frac{\sqrt{3 a}+\sqrt{a}}{\sqrt{3 a}-\sqrt{a}}=\frac{1}{\sqrt{3 a}} \ln \frac{\sqrt{3}+\sqrt{1}}{\sqrt{3}-\sqrt{1}}=\sqrt{\frac{2 \beta}{3 m}} \frac{T}{4} .
$$

So,

$$
T=4 \sqrt{\frac{m}{\alpha}} \ln \frac{\sqrt{3}+\sqrt{1}}{\sqrt{3}-\sqrt{1}} \approx 5,2678 \sqrt{\frac{m}{\alpha}} .
$$

Finally, in the third case $s^{2}=\frac{3}{4}\left(a^{2}+\frac{\alpha a}{\beta}-\frac{3}{4} \frac{\alpha^{2}}{\beta^{2}}\right)>0$.

Then expression (5) takes the form:

$$
\int_{x}^{a} \frac{d u}{\sqrt{(a-u)\left[(u+q)^{2}+s^{2}\right]}}=\sqrt{\frac{2 \beta}{3 m}} t
$$

where $q=\frac{1}{2}\left(a+\frac{3 \alpha}{2 \beta}\right)$.

The left part of (13) also reduces to an incomplete elliptic integral of the first kind. Thus, using the reference book [8], we obtain:

$$
F\left(\varphi_{*}, k_{*}\right)=\sqrt{\frac{2 \beta \gamma}{3 m}} t=\tau_{*},
$$

where $\varphi_{*}=\operatorname{arctg} \sqrt{\frac{a-x}{\gamma}} ; k_{*}=\sqrt{\frac{\gamma+q+a}{2 \gamma}} ; \gamma=\sqrt{(q+a)^{2}+s^{2}}$. 
In accordance with (14):

$$
\frac{x}{a}=1-\frac{\gamma}{a} \operatorname{tg}^{2}\left[\frac{1}{2} \operatorname{am}\left(\tau_{*}, k_{*}\right)\right] .
$$

Calculation of the period of oscillation reduces to the equal:

$$
T=4 \sqrt{\frac{3 m}{2 \beta \gamma}} F\left(\psi, k_{*}\right),
$$

where $\psi=2 \operatorname{arctg} \sqrt{a / \gamma}$.

To perform the calculations, we need to use tables of an incomplete elliptic integral of the first kind.

In the limiting case, when $\alpha \rightarrow 0, \quad$ we have $: \quad s^{2} \rightarrow \frac{3}{4} a^{2} ; \quad q \rightarrow \frac{1}{2} a ; \quad \gamma \rightarrow a \sqrt{3}$; $k_{*} \rightarrow \frac{1}{2} \sqrt{2+\sqrt{3}}=\sin 75^{\circ}, \tau_{*} \rightarrow \sqrt{\frac{2 a \beta}{\sqrt{3} m}} t$. The equals (15) and (16) are reduced according to:

$$
\begin{gathered}
\frac{x}{a}=1-\sqrt{3} \frac{1-\operatorname{cn}\left(\sqrt{\frac{2 a \beta}{\sqrt{3} m}} t, \sin 75^{\circ}\right)}{1+\operatorname{cn}\left(\sqrt{\frac{2 a \beta}{\sqrt{3} m}} t, \sin 75^{\circ}\right)} ; \\
T=4 \sqrt{\frac{\sqrt{3} m}{2 \beta \cdot a}} F\left(74,457 ; \sin 75^{\circ}\right) \approx 6,87 \sqrt{\frac{m}{a \beta}},
\end{gathered}
$$

where $\operatorname{cn}\left(\tau_{*}, \sin 75^{\circ}\right)-$ Jacobi's elliptic cosine.

Previously, such dependencies were derived in [11].

2. Free oscillations of an oscillator with a soft elasticity characteristic. Under the initial conditions a) in (2) the velocity of the oscillator is described by the expression:

$$
v=\frac{d x}{d t}=-\sqrt{\frac{2}{m}} \sqrt{\frac{\alpha}{2}\left(a^{2}-x^{2}\right)-\frac{|\beta|}{3}\left(a^{3}-x^{3}\right)}
$$

or

$$
\frac{d x}{d t}=-\sqrt{\frac{2|\beta|}{3 m}} \sqrt{(a-x)(b-x)(x-d)} .
$$

Here is a restriction $a<\alpha /|\beta|$ and the notation is used:

$$
\begin{aligned}
& b=\sqrt{\frac{1}{4}\left(\frac{3 \alpha}{2|\beta|}-a\right)^{2}+a\left(\frac{3 \alpha}{2|\beta|}-a\right)}+\frac{1}{2}\left(\frac{3 \alpha}{2|\beta|}-a\right)>0 ; \\
& d=\frac{1}{2}\left(\frac{3 \alpha}{2|\beta|}-a\right)-\sqrt{\frac{1}{4}\left(\frac{3 \alpha}{2|\beta|}-a\right)^{2}+a\left(\frac{3 \alpha}{2|\beta|}-a\right)}<0 .
\end{aligned}
$$

In the case of a soft elasticity characteristic, the initial deflection must satisfy this constraint so that free vibrations occur.

To obtain the form of displacement of an oscillator, integrate expression (17). Such a transformation gives:

$$
\int_{x}^{a} \frac{d u}{\sqrt{(a-u)(b-u)(u-d)}}=\sqrt{\frac{2|\beta|}{3 m}} t .
$$


In the left part, we have a quadrature that reduces to an incomplete elliptic integral of the first kind [8]. Therefore, expression (18) takes the form:

$$
F(\theta, \eta)=\frac{1}{2} \sqrt{\frac{2|\beta|(b-d)}{3 m}} t=\xi
$$

where $\theta=\arcsin \sqrt{\frac{(b-d)(a-x)}{(a-d)(b-x)}} ; \eta=\sqrt{\frac{a-d}{b-d}}$.

As a result, we get the following displacement equal:

$$
\frac{x}{a}=\frac{1-\frac{b}{a}\left(\frac{a-d}{b-d}\right) \operatorname{sn}^{2}(\xi, \eta)}{1-\frac{a-d}{b-d} \operatorname{sn}^{2}(\xi, \eta)},
$$

where $\operatorname{sn}(\xi, \eta)-$ Jacobi's elliptic sine.

From (19) it follows that:

$$
F(\theta *, \eta)=\frac{T}{8} \sqrt{\frac{2|\beta|(b-d)}{3 m}}
$$

where $\theta_{*}=\arcsin \sqrt{\frac{b-d}{a-d} \cdot \frac{a}{b}}$.

Then the period of fluctuations is:

$$
T=8 \sqrt{\frac{3 m}{2|\beta|(b-d)}} F\left(\theta_{*}, \eta\right) .
$$

For calculations $x / a$ and $T$, we also have to use tables of an incomplete elliptic integral of the first kind.

Let us find out what the limit transition $\beta \rightarrow 0$ gives. In this case: $b \rightarrow \infty ; d \rightarrow-a ; a-d \rightarrow 2 a$; $\eta \rightarrow 0 ; \xi \rightarrow \frac{1}{2} \sqrt{\frac{\alpha}{m}} t ; \theta_{*} \rightarrow \pi / 4 ; \operatorname{sn}(\xi, \eta) \rightarrow \sin \left(\frac{1}{2} \sqrt{\frac{\alpha}{m}} t\right)$. Equal (20) goes into (9), and equal (21) - in (11), that is, the nonlinear oscillator becomes linear.

In the limiting case, when $a \rightarrow \alpha /|\beta|$, we have: $b \rightarrow a ; d \rightarrow-0.5 a ; b / a \rightarrow 1 ; \frac{a-d}{b-d} \rightarrow 1$ and according to $(20)$ :

$$
\frac{x}{a} \rightarrow 1=\text { const }
$$

Having received the initial deviation $a=\alpha /|\beta|$, the oscillator hangs in this position of unstable equilibrium. This is how the motion of a quadratically nonlinear oscillator with a soft elasticity characteristic manifests itself in this way.

3. Oscillations caused by the given initial velocity. Using boundary conditions b) in (2), we can determine the integration constant in (3) and construct the decoupling again $x=x(t)$. But, in order not to perform such actions, we will try to adapt already constructed junctions to new initial conditions. For this it is necessary to establish a relationship between $\max x=a$ and $v_{0}$, which is connected with the search for the root of the cubic equation:

$$
a^{3}+\frac{3 \alpha}{2 \beta} a^{2}-\frac{3 m}{2 \beta} v_{0}^{2}=0
$$


In this case, one can search for roots by analytical methods [12]. But, the pain is simple, we need a solution to find, according to Newton's scheme, iterations:

$$
a_{n+1}=a_{n}-\frac{a_{n}^{3}+\frac{3 \alpha}{2 \beta} a_{n}^{2}-\frac{3 m}{2 \beta} v_{0}^{2}}{3\left(a_{n}+\frac{\alpha}{\beta}\right) a_{n}}, n=0,1,2, \ldots
$$

As an initial approximation $a_{0}$ it is advisable to take the smaller of the numbers:

$$
a_{0}=v_{0} \sqrt{\frac{m}{\alpha}} \text { and } a_{0}=\sqrt[3]{\frac{3 m}{2 \beta} v_{0}^{2}} .
$$

If, on the other hand, the oscillator has a soft elastic characteristic, then instead of (22) we have to solve the equation:

$$
a^{3}-\frac{3 \alpha}{2|\beta|} a^{2}+\frac{3 m}{2|\beta|} v_{0}^{2}=0
$$

Here, also, Newton's scheme can be convenient:

$$
a_{n+1}=a_{n}-\frac{a_{n}^{3}-\frac{3 \alpha}{2|\beta|} a_{n}^{2}+\frac{3 m}{2|\beta|} v_{0}^{2}}{3\left(a_{n}+\frac{\alpha}{|\beta|}\right) a_{n}}, n=0,1,2, \ldots,
$$

at initial approximation $a_{0}=v_{0} \sqrt{\frac{m}{\alpha}}$.

With a soft characteristic of elasticity, we have to limit the initial velocity by inequality:

$$
v_{0}<\left[v_{0}\right] \text {, }
$$

where $\left[v_{0}\right]=\frac{\alpha}{|\beta|} \sqrt{\frac{\alpha}{3 m}}$.

In the case when $v_{0}=\left[v_{0}\right]$ the amplitude of deflection $a=\alpha /|\beta|$ and oscillations is absente.

So, having calculated the deviations $x=a$, using the given value $v_{0}$, the solutions obtained above can be used to calculate the motion, by replacing in them $t$ by $(t-T / 4)$. Calculation of displacements is carried out for $(t \geq T / 4)$, and to determine $x(t)$ for smaller ones $t$, one must use the cyclicity of motion.

In order to verify the validity of the derived equals, let us consider examples for calculations.

Example 1. Let us calculate the displacements of a weakly nonlinear oscillator, to which the initial velocity $v_{0}=2.0656 \mathrm{~m} / \mathrm{s}$ is given, for, $m=2 \mathrm{~kg}, \alpha=3200 \mathrm{~N} / \mathrm{m}, \beta=6400 \mathrm{~N} / \mathrm{m}^{2}$. To find the initial deviation, let's do iteration using equal (23). To do this, we choose an initial approximation of two values: $a_{0}=v_{0} \sqrt{\frac{m}{\alpha}} \approx 0,0516 m$ and $a_{0}=\sqrt[3]{\frac{3 m}{2 \beta} v_{0}^{2}} \approx 0,126 m$.

We take less, that is $a_{0}=0.0516 \mathrm{~m}$ we accept. Its substitution in (23) and subsequent iterations yield: $a_{1}=0.050027 \mathrm{~m} ; a_{2}=0.050000 \mathrm{~m}$. In this way $a=0.05 \mathrm{~m}$. Further we find that $b \approx 0.7464$; $d \approx 0.0536 ; k \approx 0.3607 \approx \sin 21.142^{\circ} ; \tau \approx 20.609 t$. Further calculation $x / a$ is carried out according to equal (8), using linear interpolation of tabulated values in [10]. In this way, for example, we find that for $\tau=0.5266 x / a \approx 0.482$ and for $\tau=0.7051 x / a \approx 0.144$. Based on the series of calculations, we plot the graph shown in Fig. 1. The dashed part of the graph is obtained as a result of a symmetric spreading of it to the left from the ordinate axis to the intersection with the abscissa axis. This part of the graph describes the 
initial stage of the movement from position $x=0$ to position $x=a$. It is obtained purely geometrically, without corresponding calculations.

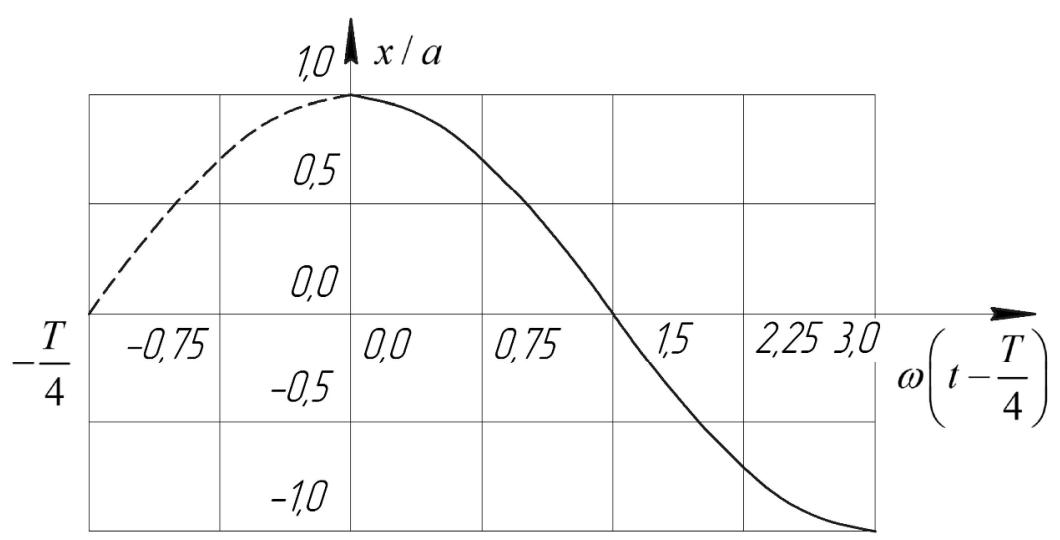

Fig. 1. Graph of oscillations of a weakly nonlinear oscillator with a rigid elastic characteristic

Example 2. In the previous example, let us consider the motion of an oscillator with a small nonlinearity. Next we analyze the case of essentially nonlinear oscillations, assuming: $m=2 \mathrm{~kg}$, $\alpha=3200 \mathrm{~N} / \mathrm{m}, \beta=32000 \mathrm{~N} / \mathrm{m}^{2}, a=0,06 \mathrm{~m}$. This initial deviation from the position $x=0$ can also be obtained by giving it the initial velocity $v_{0}=2.8397 \mathrm{~m} / \mathrm{s}$. For the specified numerical data: $s^{2}=0.001575 ; \quad q=0.105 ; \quad \gamma \approx 0.1697 ; \quad k_{*} \approx 0.9931 \approx \sin 83.242 ; \quad \gamma / a \approx 2.8284 ; \quad \tau_{*} \approx 42.5457 t$. Calculations $x / a$ are carried out according to equal (15) from the corresponding linear interpolation of tabulated data in [10]. So when $\tau_{*} \approx 0.3562$ we get $x / a \approx 0.912$, and when $\tau_{*} \approx 0.7616-x / a \approx 0.625$. The results of calculating the movements of the oscillator for others are presented in the form of a graph in Fig. 2. For comparison, the dotted line shows the graph of the motion of the linear oscillator.

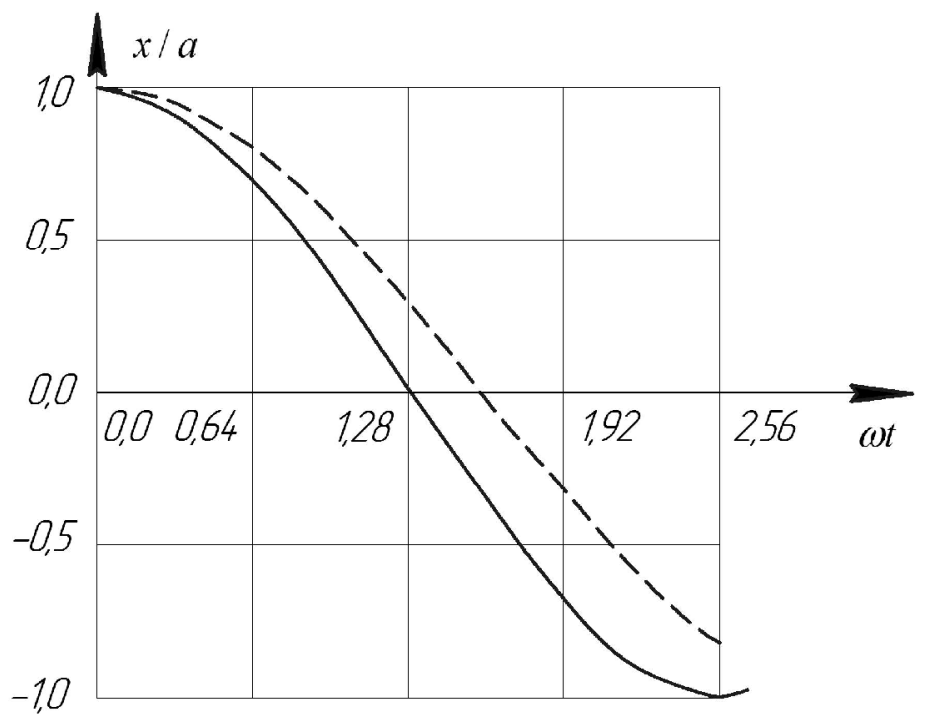

Fig. 2. Graphs of vibrations of oscillators with a rigid (solid line) and linear (nuncle line) elasticity characteristics

Example 3. Let's find out how the oscillator moves with a soft elasticity characteristic when it gives the initial velocity $v_{0}=2 \mathrm{~m} / \mathrm{s}$. For the calculation we accept: $m=1 \mathrm{~kg}, \alpha=4800 \mathrm{~N} / \mathrm{m}$, 
$|\beta|=48000 \mathrm{~N} / \mathrm{m}^{2}$. We calculate the admissible initial velocity $\left[v_{0}\right]=\frac{\alpha}{|\beta|} \sqrt{\frac{\alpha}{3 m}}=4 \mathrm{~m} / \mathrm{s}$. The initial velocity is less than the admissible one, that is, the condition for free oscillations of the oscillator is satisfied. To perform iterations according to equal (23). We calculate the initial approximation $a_{0}=v_{0} \sqrt{\frac{m}{\alpha}} \approx 0,0289 \mathrm{~m}$. Further substitution of it in (23) and iteration yields: $a_{1} \approx 0.0328 \mathrm{~m}$; $a_{2} \approx 0.0327 \mathrm{~m}$. So we accept $a=0.0327 \mathrm{~m}$. The calculated numerical data is answered by: $b \approx 0.1440 ; d \approx-0.0267 ; \eta \approx 0.5898 \approx \sin 36.144^{\circ} ; \xi \approx 36.9432 t$. Further substitution of them into the equal (20), using the table of an incomplete elliptic integral of the first kind, determines $x / a$. So, for $\xi \approx 0.1748$ we get $x / a \approx 0.964$, and for $\xi \approx 0.5318-x / a \approx 0.676$. As a result, we plot the displacement graph shown in Fig. 3.

The dotted part of the graph is obtained in a purely geometric way, namely, by extending the graph to the left symmetrically about the ordinate axis before crossing with the abscissa axis.

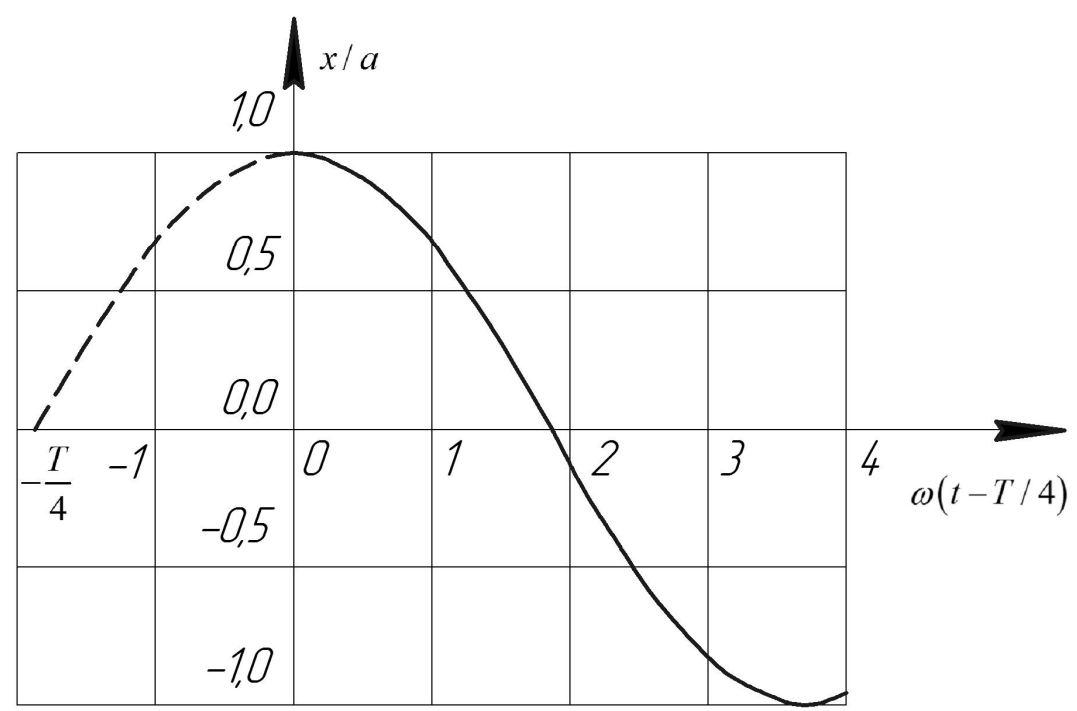

Fig. 3. Displacement of oscillator with a soft elasticity characteristic

\section{Conclusions}

1. Analytical solutions of the Cauchy problem for a nonlinear differential equation of motion are expressed in terms of Jacobi periodic elliptic functions.

2. The derived equals have the ability to calculate the displacements and period of oscillations of a quadratically nonlinear oscillator, with both rigid and soft elastic characteristics. The reason for the oscillations may be either the initial deviation of the oscillator from the stable equilibrium position or the initial velocity in this position.

3. In the case of a soft elasticity characteristic, free oscillations are possible only if the specified limitations on the initial deviation and on the initial velocity are observed.

\section{References}

[1] A. A. Larin, Ocherki istorii razvitija terii mehanicheskih kolebanij [Essays on the History of Development of the Theory of Mechanical Oscillations]. Sevastopol', Ukraine: Veber Publ., 2013. (In Russian).

[2] K. V. Avramov and Ju.V. Mihlin, Nelinejnaja dinamika uprugih sistem. T. 1. Modeli, metody, javlenija [Nonlinear Dynamics of Elastic Systems. Vol. 1. Models, methods, phenomena]. Moscow, Russia: Institut komp'juternyh issledovanij Publ., 2010. (In Russian). 
[3] Ju. A. Mitropol's'kij, Izbrannye trudy v 2-h tomah [Selected works in 2 volumes]. Kyiv, Ukraine: Naukova dumka Publ., 2012. (In Russian).

[4] P. Ya. Pukach, Yakisni metody doslidzhennia neliniinykh kolyvalnykh system [Qualitative methods for investigating nonlinear oscillation systems]. Lviv, Ukraine: Lviv Polytechnic Publ., 2014. (In Ukrainian).

[5] V. M. Shatohin, Analiz i parametricheskij sintez nelinejnyh silovyh peredach mashin [Analysis and parametric synthesis of nonlinear power transmission of machines]. Kharkiv, Ukraine: NTU "HPI" Publ., 2008. (In Russian).

[6] V. P. Olshanskiy and S. V. Olshanskiy, Metod VBK v raschetah nestacionarnyh kolebanij oscilljatorov [The VBK method in calculations of non-stationary oscillations of oscillators]. Kharkiv, Ukraine: Mis'kdruk Publ., 2014. (In Russian).

[7] V. P. Olshanskiy, S. V. Olshanskiy and L. M. Tishchenko, Dynamika dysypatyvnykh ostsyliatoriv [Dynamics of dissipative oscillators]. Kharkiv, Ukraine: Mis'kdruk Publ., 2016. (In Ukrainian).

[8] I. S. Gradshtejn and I. M. Ryzhik, Tablicy integralov, summ, rjadov i proizvedenij [Tables of integrals, sums, series, and products]. Moscow, Russia: Nauka Publ., 1962. (In Russian).

[9] A. Abramovic and I. Stigan, Spravochnik po special'nym funkcijam (s formulami, grafikami $i$ matematicheskimi tablicami) [Handbook of special functions (with formulas, graphs and mathematical tables)]. Moscow, Russia: Nauka Publ., 1979. (In Russian).

[10] E. Janke, F. Jemde and F. Lesh, Special'nye funkcii [Special functions]. Moscow, Russia: Nauka Publ., 1977. (In Russian).

[11] V. P. Olshanskiy et al., "Pro kolyvannia ostsyliatora z kvadratychno-neliniinoiu zhorstkistiu" ["About fluctuation of oscillator with quadratic nonlinear stiffness"], Tekhnichnyi servis ahropromyslovoho, lisovoho ta transportnoho kompleksiv [Technical service of agriculture, forestry and transport systems], no. 8, pp. 177-185, 2017. (in Ukrainian).

[12] G. Korn and T. Korn, Spravochnik po matematike (dlja nauchnyh rabotnikov i inzhenerov) [Handbook of Mathematics (for scientists and engineers)]. Moscow, Russia: Nauka Publ., 1974. (In Russian). 